\title{
The Upper and Lower Functions Method for Second Order Systems
}

\author{
A. Ja. Lepin and F. Zh. Sadyrbaev
}

\begin{abstract}
Two-point boundary value problems for $m$-dimensional second order systems are considered. The method of upper and lower functions is applied to problems of the Dirichlet type and problems with nonlinear boundary conditions. The conditions on upper and lower functions are substantially relaxed comparing with the classical $C^{2}$-class and properties of them are studied for systems with monotone in $x$ right sides. Consequences for even order differential equations with mixed monotonicities are given.
\end{abstract}

Keywords: Monotone iterative techniques, upper and lower functions, maximal and minimal solutions, second order systems

AMS subject classification: $34 \mathrm{~B} 15$

\section{Introduction}

For $a, b \in \mathbb{R}$ with $a<b$ set $I=[a, b]$. Consider the scalar second order differential equation

$$
x^{\prime \prime}=f\left(t, x, x^{\prime}\right)
$$

and its short form

$$
x^{\prime \prime}=f(t, x) \quad(t \in I)
$$

where the right sides are continuous functions. Let the boundary conditions be in the Dirichlet form

$$
\left.\begin{array}{l}
x(a)=A \\
x(b)=B
\end{array}\right\} .
$$

Well known result (see [2: Chapter 1/Theorem 1.5.1] or [11: Chapter 3, $\S 1 /$ Theorem 1]) in the theory of two-point boundary value problems states that if there exist functions $\alpha, \beta \in C^{(2)}(I, \mathbb{R})$ (which are referred to usually as lower and upper functions) such that

(C0) $\alpha^{\prime \prime}(t) \geq f(t, \alpha(t))$ and $\beta^{\prime \prime}(t) \leq f(t, \beta(t))$

(C1) $\alpha(t) \leq \beta(t)$ for all $t \in I$

(C2) $\alpha(a) \leq A \leq \beta(a)$ and $\alpha(b) \leq B \leq \beta(b)$,

Both authors: Univ. of Latvia, Inst. Math. \& Comp. Sci., LV-1459 Riga, Latvia.

Research supported by Latvian Council of Science, project 01.0356.

felix@cclu.lv 
then problem $(2)$ - (3) has a solution $x \in C^{(2)}(I, \mathbb{R})$ such that

$$
\alpha(t) \leq x(t) \leq \beta(t) \quad(t \in I)
$$

A solution to problem (1), (3) exists also under some additional conditions which are referred to usually as Bernstein-Nagumo type conditions (see [2: Chapter 1, Sec. 1.4] or [7: Chapter XII, Part 2]).

The upper and lower functions $\beta$ and $\alpha$ exist, for example, if $f(t, x) \rightarrow \pm \infty$ as $x \rightarrow \pm \infty$, respectively. Then $\beta$ can be chosen as a large enough constant $\beta$ and $\alpha=-\beta$. So, generally, if $f(t, x)$ increases with respect to $x$, the existence of $\alpha$ and $\beta$ can be expected. It can be shown easily that for any arbitrary continuous function $f$ upper and lower functions can be constructed always, on small enough intervals. Indeed, consider the Cauchy problem for equation (2) $x(a)=A$ and $x^{\prime}(a)= \pm A_{1}$. For any positive $A_{1}$, a solution $x\left(t ; A_{1}\right)$ is greater than $x\left(t ;-A_{1}\right)$ on a small enough interval $(a, \tau]$. So, for any $B \in\left[x\left(b ;-A_{1}\right), x\left(b ; A_{1}\right)\right]$ where $a<b<\tau$, a solution to problem (2) - (3) exists, since $\beta(t)=x\left(t ; A_{1}\right)$ and $\alpha(t)=x\left(t ;-A_{1}\right)$ satisfy conditions $(\mathrm{C} 0)$ - (C2).

The method of upper and lower functions is effective only if $\alpha$ and $\beta$ can be found which are not identically equal (then they coincide with a solution). Otherwise the process of finding $\alpha$ and $\beta$ is not easier than the process of finding a solution itself.

It can be shown that for the problem $x^{\prime \prime}=-k^{2} x, x(0)=x(1)=0$ distinct functions $\alpha$ and $\beta$ exist only for $|k| \leq \pi$. Indeed, if $\alpha$ and $\beta$ exist, then the non-negative non-trivial difference $u=\beta-\alpha$ satisfies the equation $u^{\prime \prime}=-k^{2} u-\varepsilon(t)$ where $\varepsilon$ is a non-negatively valued continuous function. Following [11: Chapter 3, §2] we multiply both sides by $\sin \pi t$ and integrate twice over the interval $[0,1]$. The resulting identity

$$
\pi(u(0)+u(1))+\left(k^{2}-\pi^{2}\right) \int_{0}^{1} \sin \pi t u(t) d t=-\int_{0}^{1} \sin \pi t \varepsilon(t) d t
$$

is impossible if $k^{2}>\pi^{2}$. Hence, equations with right side $f(t, x)$, which decreases in $x$, generally allow for application of the upper and lower functions method on small intervals only. The situation is analogous for second order systems. It is worthy to mention that systems of form (2) with right sides decreasing in $x$ appear in applications, since they describe various oscillation phenomena.

The aim of this paper is to investigate the possibility of application of the upper and lower functions method to the existence of solutions for second order systems with right sides $f(t, x)$ decreasing in $x$. In Section 2 we provide a general existence result for the Dirichlet problem. In Section 3 several properties of the lower and upper functions $\alpha$ and $\beta$ are considered for the case of decreasing right sides. In Section 4 boundary value problems with nonlinear boundary conditions are considered and existence results are proved. In Section 5 systems without monotonicity assumption are treated as well as Bernstein-Nagumo type conditions for systems containing the derivative $x^{\prime}$ in the right side. Definitions of the lower and upper functions $\alpha$ and $\beta$, suitable for this case, and existence results are presented. The final Section 6 is devoted to even order scalar differential equations with mixed monotonicities. 


\section{General existence result}

Consider a system

$$
x^{\prime \prime}=f(t, x) \quad\left(t \in I, x \in \mathbb{R}^{m}\right)
$$

where $I=[a, b]$ and $f: I \times \mathbb{R}^{m} \rightarrow \mathbb{R}^{m}$ satisfies the Carathéodory conditions, that is:

(i) For $t$ fixed, $f(t, \cdot)$ is a continuous function.

(ii) For $x$ fixed, $f(\cdot, x)$ is Lebesque-measurable.

(iii) For any $M>0$ there exists $g \in L_{1}\left(I,[0, \infty)^{m}\right)$ such that $|f(t, x)| \leq g(t)$ for $t \in[a, b]$ and $\left|x_{i}\right|<M(i=1, \ldots, m)$.

Let the boundary conditions be of the form

$$
\left.\begin{array}{l}
x(a)=A \\
x(b)=B
\end{array}\right\} \quad\left(A, B \in \mathbb{R}^{m}\right) .
$$

Our main assumption in the sequel is

(M) For any $t \in I, f(t, \cdot)$ is non-increasing.

Definition 2.1. Functions $\alpha, \beta: I \rightarrow \mathbb{R}^{m}$ will be called lower and upper functions for system (4) if they satisfy the Lipschitz condition and for any points $t_{1} \in(a, b)$ and $t_{2} \in\left(t_{1}, b\right)$, in which first order derivatives exist, the inequalities

$$
\left.\begin{array}{l}
\alpha^{\prime}\left(t_{2}\right)-\alpha^{\prime}\left(t_{1}\right) \geq \int_{t_{1}}^{t_{2}} f(t, \alpha(t)) d t \\
\beta^{\prime}\left(t_{2}\right)-\beta^{\prime}\left(t_{1}\right) \leq \int_{t_{1}}^{t_{2}} f(t, \beta(t)) d t
\end{array}\right\}
$$

hold, respectively.

It seems that inequalities (6) appeared for the first time in the work by I. Kiguradze [9]. Various relaxations of differential properties of $\alpha$ and $\beta$ were obtained in the papers $[1,3,5,6,14]$ (see also the survey [4] and the references therein). In the case of a scalar equation (4) inequalities (6) mean that $\alpha^{\prime}$ and $\beta^{\prime}$, which evidently are functions of bounded variation, when decomposed to the sum of an absolute continuous function and a singular one, have monotone singular parts, namely, non-decreasing for $\alpha^{\prime}$ and non-increasing for $\beta^{\prime}$. Moreover, the differences $\alpha^{\prime}(t)-\int^{t} f(t, \alpha(t)) d t$ and $\beta^{\prime}(t)-\int^{t} f(t, \beta(t)) d t$ are non-decreasing and non-increasing in $t$, respectively. For discussion, proofs and references one may consult $[3-5,12,14]$.

Denote

$$
D=\left\{(t, x) \in I \times \mathbb{R}^{m}: \alpha(t) \leq x \leq \beta(t)\right\} .
$$

Our main existence result is the following 
Theorem 2.1 (Ceneral Existence Theorem). Let condition (M) be satisfied and let upper and lower functions $\alpha$ and $\beta$ in the sense of inequalities (6) exist such that

(A1) $\alpha(t) \leq \beta(t)$ for all $t \in I$.

(A2) $\alpha(a) \leq A \leq \beta(a)$ and $\alpha(b) \leq B \leq \beta(b)$.

Then there exists a solution $x$ of problem (4) - (5) such that

$$
(t, x(t)) \in D \quad(t \in I) .
$$

Proof. Consider the modified equation

$$
x^{\prime \prime}=f(t, X)+\Delta_{1}(x, \beta)-\Delta_{2}(\alpha, x)
$$

where

$$
\begin{aligned}
X & =\left(X_{1}, \ldots, X_{m}\right) \text { with } X_{i}=\delta\left(\alpha_{i}(t), x_{i}, \beta_{i}(t)\right) \quad(i=1, \ldots, m) \\
\delta(u, z, v) & = \begin{cases}v & \text { if } z>v \\
z & \text { if } u \leq z \leq v \\
u & \text { if } z<u\end{cases} \\
\Delta_{1} & =E_{m} \cdot \operatorname{col}\left(\delta\left(0, x_{1}-\beta_{1}(t), 1\right), \ldots, \delta\left(0, x_{m}-\beta_{m}(t), 1\right)\right) \\
\Delta_{2} & =E_{m} \cdot \operatorname{col}\left(\delta\left(0, \alpha_{1}(t)-x_{1}, 1\right), \ldots, \delta\left(0, \alpha_{m}(t)-x_{m}, 1\right)\right)
\end{aligned}
$$

with $E_{m}$ the unity matrix of order $m$. Problem $(8),(5)$ has a solution $x$ since the right side in equation (8) is globally bounded and satisfies the Carathéodori conditions, and the homogeneous problem $x^{\prime \prime}=0, x(a)=x(b)=0$ has the trivial solution only (see, for example, [11: Chapter 2, §2/ Existence Theorem]).

To prove the theorem it suffices now to show that estimate $(7)$ is valid. Suppose $(7)$ does not hold. To be specific, suppose $x_{1}(t)>\beta_{1}(t)$ on some subinterval of $I$. Denote $u_{1}=x_{1}-\beta_{1}$. If

$$
u_{1}\left(t_{0}\right)=\max _{I} u_{1}(t)=\max _{I}\left\{x_{1}(t)-\beta_{1}(t)\right\}
$$

then in any arbitrarily small vicinity of $t_{0}$ there exist $t_{1}<t_{0}$ and $t_{2}>t_{0}$ such that the derivatives $u_{1}^{\prime}\left(t_{1}\right)$ and $u_{1}^{\prime}\left(t_{2}\right)$ exist and $u_{1}^{\prime}\left(t_{2}\right)-u_{1}^{\prime}\left(t_{1}\right) \leq 0$. On the other hand,

$$
\begin{aligned}
u_{1}^{\prime}\left(t_{2}\right)-u_{1}^{\prime}\left(t_{1}\right)= & \int_{t_{1}}^{t_{2}} f_{1}\left(s, \beta_{1}(s), X_{2}(s), \ldots, X_{m}(s)\right) d s \\
& +\int_{t_{1}}^{t_{2}} \delta\left(0, x_{1}(s)-\beta_{1}(s), 1\right) d s-\int_{t_{1}}^{t_{2}} f_{1}(s, \beta(s)) d s \\
& >0
\end{aligned}
$$

since, in view of the monotonicity of $f(t, x)$ with respect to the variables $x_{2}, \ldots, x_{m}$ and the definition of $X_{i}$,

$$
f_{1}\left(s, \beta_{1}(s), X_{2}(s), \ldots, X_{m}(s)\right) \geq f_{1}(s, \beta(s))
$$


The contradiction obtained proves that $x_{1}(t) \leq \beta_{1}(t)$ for all $t \in I$.

It can be shown similarly that $x_{1} \geq \alpha_{1}$. Indeed, if the difference $v_{1}=x_{1}-\alpha_{1}$ has a negative minimum, then in some vicinity of the point of minimum there exist $t_{1}$ and $t_{2}$ such that $t_{1}<t_{2}$ and $v_{1}^{\prime}\left(t_{2}\right)-v_{1}^{\prime}\left(t_{1}\right) \geq 0$ as well as $v_{1}(t)<0$ for all $t \in\left[t_{1}, t_{2}\right]$. On the other hand,

$$
\begin{aligned}
v_{1}^{\prime}\left(t_{2}\right)-v_{1}^{\prime}\left(t_{1}\right)= & \int_{t_{1}}^{t_{2}} f_{1}\left(s, \alpha_{1}(s), X_{2}(s), \ldots, X_{m}(s)\right) d s \\
& -\int_{t_{1}}^{t_{2}} \delta\left(0, \alpha_{1}(s)-x_{1}(s), 1\right) d s-\int_{t_{1}}^{t_{2}} f_{1}(s, \alpha(s)) d s \\
< & 0
\end{aligned}
$$

since, in view of the monotonicity of $f(t, x)$ with respect to the variables $x_{2}, \ldots, x_{m}$ and the definition of $X_{i}$,

$$
f_{1}\left(s, \alpha_{1}(s), X_{2}(s), \ldots, X_{m}(s)\right) \leq f_{1}(s, \alpha(s)) .
$$

The contradiction obtained proves that $x_{1}(t) \geq \alpha_{1}(t)$ for all $t \in I$. The proofs for the components $x_{2}, \ldots, x_{m}$ are similar

Remark 2.1. In the proof for the component $x_{1}$ monotonicity of $f_{1}(t, x)$ with respect to the variables $x_{2}$ to $x_{m}$ only was exployed. Similarly, proof for the component $x_{i}$ uses monotonicity of $f_{i}(t, x)$ with respect to the variables $x_{1}, \ldots, x_{i-1}, x_{i+1}, \ldots, x_{m}$. Therefore, the result of the General Existence Theorem is valid for functions $f(t, x)$ which are monotone in non-diagonal variables only (that is, in $x_{j}(j \neq i)$ for $\left.f_{i}\right)$.

\section{Properties of $\alpha$ and $\beta$}

3.1 Convexity. It follows from (6), the inequality $\alpha \leq \beta$ and the monotonicity condition $(\mathrm{M})$ that the difference $u=\beta-\alpha$ satisfies the relation

$$
u^{\prime}\left(t_{2}\right)-u^{\prime}\left(t_{1}\right) \leq \int_{t_{1}}^{t_{2}}(f(t, \beta(t))-f(t, \alpha(t))) d t \leq 0 \quad\left(t_{1}<t_{2}\right) .
$$

Thus $u^{\prime}$ is a non-increasing function and the difference $\beta-\alpha$ is a concave function.

Remark 3.1. A usual assumption about $\alpha$ and $\beta$ for the Neumann problem

$$
\left.\begin{array}{rl}
x^{\prime \prime} & =f(t, x) \quad(\alpha \leq x \leq \beta) \\
x^{\prime}(a) & =A, x^{\prime}(b)=B
\end{array}\right\}
$$

is that

$$
\left.\begin{array}{l}
\alpha^{\prime}(a) \geq A \geq \beta^{\prime}(a) \\
\alpha^{\prime}(b) \leq B \leq \beta^{\prime}(b)
\end{array}\right\}
$$

This assumption is essential as the example of the problem $x^{\prime \prime}=-x, x^{\prime}(0)=x^{\prime}(\pi)=1$ shows. Indeed, $\beta(t)=\sin t$ and $\alpha(t)=-\sin t$ are upper and lower functions with 
$\beta(t)>\alpha(t)$ on the interval $(0, \pi)$, but conditions (10) are not fulfilled and the problem has not a solution.

It follows from (10) that $u^{\prime}(a) \leq 0$ and $u^{\prime}(b) \geq 0$. Together with the concavity of $u$ this means that $u=$ const. Let $y$ and $z$ be solutions of the equation $x^{\prime \prime}=f(t, x)$ satisfying the boundary conditions

$$
\begin{aligned}
& y(a)=\alpha(a) \\
& y(b)=\alpha(b)
\end{aligned} \quad \text { and } \quad \begin{aligned}
& z(a)=\beta(a) \\
& z(b)=\beta(b),
\end{aligned}
$$

respectively, and such that $\alpha(t) \leq y(t) \leq \beta(t)$ and $y(t) \leq z(t) \leq \beta(t)$ for all $t \in I$. Such solutions exist, by the General Existence Theorem. The difference $v=z-y$ satisfies

$$
\begin{aligned}
& v^{\prime}(a)=z^{\prime}(a)-y^{\prime}(a) \leq \beta^{\prime}(a)-\alpha^{\prime}(a)=0 \\
& v^{\prime}(b)=z^{\prime}(b)-y^{\prime}(b) \geq \beta^{\prime}(b)-\alpha^{\prime}(b)=0 .
\end{aligned}
$$

In view of condition (M) and (11) - (12), $v=$ const $=u$ and hence $\alpha=y$ and $\beta=z$. This means that $\alpha$ and $\beta$ must be solutions of equation (4). The problem of finding $\alpha$ and $\beta$ for the Neumann problem is therefore not easier than the solution of equation $(4)$.

3.2 Converging iterations. Suppose that $\alpha$ and $\beta$ given satisfy conditions (A1) and (A2). Consider the set $\Omega(\alpha, \beta)$ of solutions to problem (4)- (5) satisfying estimate (7). We say that $u$ and $v$ are a minimal and maximal solutions of problem (4)- (5) if $u(t) \leq x(t)$ and $v(t) \geq x(t)$ for all $t \in I$, respectively, for any other solution $x \in \Omega(\alpha, \beta)$ of the problem.

Consider now the following construction. Set $y_{1}=\alpha$. A solution to the problem

$$
\left.\begin{array}{rl}
y_{n+1}^{\prime \prime} & =f\left(t, y_{n}\right) \\
y_{n+1}(a) & =A, y_{n+1}(b)=B
\end{array}\right\} \quad(n \in \mathbb{N})
$$

is given by the formula

$$
\begin{aligned}
y_{n+1}(t)= & A+\frac{B-A}{b-a}(t-a) \\
& -\frac{1}{b-a} \int_{a}^{t}(b-t)(s-a) f\left(s, y_{n}(s)\right) d s \\
& -\frac{1}{b-a} \int_{t}^{b}(b-s)(t-a) f\left(s, y_{n}(s)\right) d s .
\end{aligned}
$$

This process yields a sequence $\left\{y_{n}\right\}$. Similarly, a sequence $\left\{z_{n}\right\}$ can be obtained starting with $z_{1}=\beta$ and solving the problem

$$
\left.\begin{array}{rl}
z_{n+1}^{\prime \prime} & =f\left(t, z_{n}\right) \\
z_{n+1}(a) & =A, z_{n+1}(b)=B
\end{array}\right\} \quad(n \in \mathbb{N}) .
$$

The following result is valid. 
Theorem 3.1. Let condition (M) be satisfied. The sequences $\left\{y_{n}\right\}$ and $\left\{z_{n}\right\}$ consist of lower and upper functions, respectively, ordered by

$$
y_{1} \leq y_{2} \leq \ldots \leq z_{2} \leq z_{1}
$$

Moreover, the sequences $\left\{y_{n}\right\}$ and $\left\{z_{n}\right\}$ converge to a minimal and maximal solution $y$ and $z$ of problem $(4)-(5)$, respectively.

Proof. Let us show that $y_{2}(t) \geq y_{1}(t)$ for all $t \in I$. Inequality $(6)_{1}$ and

$$
y_{2}^{\prime}\left(t_{2}\right)-y_{2}^{\prime}\left(t_{1}\right)=\int_{t_{1}}^{t_{2}} f\left(t, y_{1}(t)\right) d t \quad\left(t_{2}>t_{1}\right)
$$

together imply that $u^{\prime}\left(t_{2}\right)-u^{\prime}\left(t_{1}\right) \leq 0$ for the difference $u=y_{2}-y_{1}$. Therefore, $u^{\prime}$ is a non-increasing function. Then, in view of $u(a) \geq 0$ and $u(b) \geq 0, u(t) \geq 0$ for all $t \in I$. It follows from (13) that $y_{2}$ is a lower function. Similarly the estimate $y_{2} \leq \beta$ can be obtained. A monotone and bounded sequence $\left\{y_{n}\right\}$ has a limit $y$. It follows from (14) that $y$ is a solution to the Dirichlet problem (4) - (5) and (7) holds.

Let us show that $y$ is a minimal solution of system (4) - (5). Suppose $x$ is another solution of this problem. Then

$$
\begin{aligned}
x(t)= & A+\frac{B-A}{b-a}(t-a) \\
& -\frac{1}{b-a} \int_{a}^{t}(b-t)(s-a) f(s, x(s)) d s \\
& -\frac{1}{b-a} \int_{t}^{b}(b-s)(t-a) f(s, x(s)) d s .
\end{aligned}
$$

Subtracting (14) at $n=1$ from (15) gives

$$
\begin{aligned}
x(t)-y_{2}(t)= & -\frac{1}{b-a} \int_{a}^{t}(b-t)(s-a)\left[f(s, x(s))-f\left(s, y_{1}(s)\right)\right] d s \\
& -\frac{1}{b-a} \int_{t}^{b}(b-s)(t-a)\left[f(s, x(s))-f\left(s, y_{1}(s)\right)\right] d s \\
\geq & 0 .
\end{aligned}
$$

Therefore, $x \geq y_{2}$. Similarly a proof of the estimate $x \geq y_{n}$ can be conducted. Thus $x \geq y$. Properties of $\left\{z_{n}\right\}$ and $z$ can be proved in a similar manner

Remark 3.2. The proof of Theorem 3.1 provides an approximate method for solution of problem (4) - (5). 


\section{Nonlinear boundary conditions}

Denote by $S$ the set of all solutions of equation (4) which satisfy (7). Consider the problem

$$
\left.\begin{array}{rl}
x^{\prime \prime} & =f(t, x) \\
H_{a} x & =H_{b} x=0
\end{array}\right\} \quad(\alpha \leq x \leq \beta)
$$

where $H_{a}, H_{b}: A C^{(1)}\left([a, b], \mathbb{R}^{m}\right) \rightarrow \mathbb{R}^{m}$ are functionals continuous in the $C^{(1)}$-norm. By $A C^{(1)}$ we mean the set of functions with absolutely continuous first order derivative. The $C^{(1)}$-norm is defined by

$$
\|x\|=\max _{1 \leq i \leq m}\left\{\max _{a \leq t \leq b}\left|x_{i}(t)\right|+\max _{a \leq t \leq b}\left|x_{i}^{\prime}(t)\right|\right\} .
$$

In what follows we need the following conditions. For any $x \in S$ and $i=1, \ldots, m$,

$$
H_{a i} x\left\{\begin{array} { l l } 
{ \leq 0 } & { \text { if } x _ { i } ( a ) = \alpha _ { i } ( a ) } \\
{ \geq 0 } & { \text { if } x _ { i } ( a ) = \beta _ { i } ( a ) }
\end{array} \quad \text { and } \quad H _ { b i } x \left\{\begin{array}{ll}
\leq 0 & \text { if } x_{i}(b)=\alpha_{i}(b) \\
\geq 0 & \text { if } x_{i}(b)=\beta_{i}(b)
\end{array}\right.\right.
$$

Remark 4.1. If the functionals $H_{a}$ and $H_{b}$ have the form $H_{a i} x=H_{a i} x_{i}$ and $H_{b i} x=H_{b i} x_{i} \quad(i=1, \ldots, m)$, then the existence of a solution to problem (16) can be proved making use of the results in [12: Chapter 3]. The proof is similar to that of Theorem 3.1 .

Consider the problem

$$
\left.\begin{array}{rl}
x^{\prime \prime} & =f(t, x) \\
x(a) & =\phi_{a}(x), x(b)=\phi_{b}(x)
\end{array}\right\} \quad(\alpha \leq x \leq \beta)
$$

where $\phi_{a}, \phi_{b}: A C^{(1)}\left([a, b], \mathbb{R}^{m}\right) \rightarrow \mathbb{R}^{m}$ are functionals continuous in the $C^{(1)}$-norm. To formulate the next result we need the condition

$$
\begin{aligned}
& \alpha_{i}(\tau) \leq \min \left\{\phi_{\tau i}(x): x \in S \text { and } x_{i}(\tau)=\alpha_{i}(\tau)\right\} \\
& \beta_{i}(\tau) \geq \max \left\{\phi_{\tau i}(x): x \in S \text { and } x_{i}(\tau)=\beta_{i}(\tau)\right\}
\end{aligned}
$$

for any $\tau \in\{a, b\}$ and $i=1, \ldots, m$.

Theorem 4.1. Problem (18) is solvable if conditions (19) are fulfilled.

Proof. Consider the modified problem

$$
\left.\begin{array}{rl}
x^{\prime \prime} & =f(t, X)+\Delta_{1}(x, \beta)-\Delta_{2}(\alpha, x) \\
x(a) & =\delta\left(\alpha(a), \phi_{a}(x), \beta(a)\right) \\
x(b) & =\delta\left(\alpha(b), \phi_{b}(x), \beta(b)\right)
\end{array}\right\}
$$

where the components of the vector function $\delta$ are scalar functions, defined in Section 2 , as well as $\Delta_{1}$ and $\Delta_{2}$, and $X$ is defined in (9). The modified problem has a solution $x$, since the homogeneous problem $x^{\prime \prime}=0, x(a)=x(b)=0$ has the trivial solution only 
[11: Chapter 3, $\S 1 /$ Theorem 1]. Let us show that $x$ is a solution of problem (18). It follows from the boundary conditions that $\alpha(a) \leq x(a) \leq \beta(a)$ and $\alpha(b) \leq x(b) \leq \beta(b)$. The proof of the estimate $\alpha \leq x \leq \beta$ is a mere repetition of the arguments used in the proof of Theorem 2.1.

Let us prove that $x_{1}(a)=\phi_{a 1}(x)$. This is evident in the case of $\alpha_{1}(a)<x_{1}(a)<$ $\beta_{1}(a)$. Consider the case of $x_{1}(a)=\alpha_{1}(a)$. This is possible only if $\phi_{a 1}(x) \leq \alpha_{1}(a)$. However, condition (19) ${ }_{1}$ imply $\alpha_{1}(a) \leq \phi_{a 1}(x)$. Hence $x_{1}(a)=\alpha_{1}(a)=\phi_{a 1}(x)$. The equalities $x(a)=\phi_{a}(x)$ and $x(b)=\phi_{b}(x)$ can be obtained in the same fashion

Remark 4.2. 1. Analogous results for symmetric first order systems were obtained in [10]. 2. If $H_{a}$ and $H_{b}$ in (16) satisfy conditions (17), then $\phi_{a}(x)=x(a)-H_{a}(x)$ and $\phi_{b}(x)=x(b)-H_{b}(x)$ satisfy conditions (19). 3. If for any $A$ and $B$ satisfying condition (A2) the Dirichlet problem (4) - (5) has a unique solution $x$ with $\alpha \leq x \leq \beta$, then $S$ is homeomorphic to the $2 m$-dimensional cube $\left[\alpha_{1}(a), \beta_{1}(a)\right] \times \cdots \times\left[\alpha_{m}(b), \beta_{m}(b)\right]$ and problem (16) is solvable provided that conditions (17) are fulfilled.

\section{General systems}

In this section we do not assume that $f(t, x)$ is monotone.

Definition 5.1. Functions $\alpha, \beta:[a, b] \rightarrow \mathbb{R}^{m}$ such that $\alpha \leq \beta$ will be called lower and upper functions if they satisfy the Lipschitz condition and for any points $t_{1}, t_{2} \in$ $(a, b)$ with $t_{1}<t_{2}$, in which first order derivatives exist, and for any $x \in A C^{(1)}\left(I, \mathbb{R}^{m}\right)$ the inequalities

$$
\left.\begin{array}{l}
\alpha_{i}^{\prime}\left(t_{2}\right)-\alpha_{i}^{\prime}\left(t_{1}\right) \geq \int_{t_{1}}^{t_{2}} f_{i}\left(t, X_{\alpha_{i}}(t)\right) d t \\
\beta_{i}^{\prime}\left(t_{2}\right)-\beta_{i}^{\prime}\left(t_{1}\right) \leq \int_{t_{1}}^{t_{2}} f\left(t, X_{\beta_{i}}(t)\right) d t
\end{array}\right\} \quad(1=1, \ldots, m)
$$

hold, respectively, where

$$
X_{\gamma_{i}}=\left(X_{1}, \ldots, X_{i-1}, \gamma_{i}, X_{i+1}, \ldots, X_{m}\right), X_{i}=\delta\left(\alpha_{i}, x_{i}, \beta_{i}\right) .
$$

For classical $\alpha, \beta \in C^{(2)}(I)$ the new definition requires that the differential inequalities

$$
\left.\begin{array}{rl}
\alpha_{i}^{\prime \prime}(t) & \geq f\left(t, X_{\alpha_{i}}\right) \\
\beta_{i}^{\prime \prime}(t) & \leq f\left(t, X_{\beta_{i}}\right)
\end{array}\right\} \quad(i=1, \ldots, m)
$$

hold for any $C^{(2)}$-function $x$. In the case of $f(t, x)$ being monotone (non-increasing), the inequalities $\alpha^{\prime \prime}(t) \geq f(t, \alpha(t))$ and $\beta^{\prime \prime}(t) \leq f(t, \beta(t))$ imply $(22)$.

Theorem 5.1. Let $\alpha$ and $\beta$ exist in the sense of (20) and let conditions (A1) and (A2) be satisfied. Then problem (4) - (5) is solvable.

We omit the proof since it consists of repetition of the proof of Theorem 2.1 with evident changes.

Consider the general system

$$
x^{\prime \prime}=f\left(t, x, x^{\prime}\right) \quad(a \leq t \leq b)
$$

where the right side is supposed to satisfy the Carathéodory conditions. 
Definition 5.2. Functions $\alpha, \beta:[a, b] \rightarrow \mathbb{R}^{m}$ such that $\alpha \leq \beta$ will be called lower and upper functions for system (23) if they satisfy the Lipschitz condition and for any $t_{1}, t_{2} \in(a, b)$ with $t_{1}<t_{2}$, in which their first order derivatives exist, and for any $x \in A C^{(1)}\left(I, \mathbb{R}^{m}\right)$ the inequalities

$$
\left.\begin{array}{l}
\alpha_{i}^{\prime}\left(t_{2}\right)-\alpha_{i}^{\prime}\left(t_{1}\right) \geq \int_{t_{1}}^{t_{2}} f_{i}\left(t, X_{\alpha_{i}}(t), X_{\alpha_{i}}^{\prime}(t)\right) d t \\
\beta_{i}^{\prime}\left(t_{2}\right)-\beta_{i}^{\prime}\left(t_{1}\right) \leq \int_{t_{1}}^{t_{2}} f\left(t, X_{\beta_{i}}(t), X_{\beta_{i}}^{\prime}(t)\right) d t
\end{array}\right\} \quad(i=1, \ldots, m)
$$

hold, respectively, where $X_{\gamma_{i}}$ are defined in (21) and

$$
X_{\gamma_{i}}^{\prime}=\left(x_{1}^{\prime}(t), \ldots, x_{i-1}^{\prime}(t), \gamma_{i}^{\prime}(t), x_{i+1}^{\prime}(t), \ldots, x_{m}^{\prime}(t)\right) .
$$

Consider the problem

$$
\left.\begin{array}{rl}
x^{\prime \prime} & =f\left(t, x, x^{\prime}\right) \\
x(a) & =\phi_{a}(x), x(b)=\phi_{b}(x)
\end{array}\right\} \quad(\alpha \leq x \leq \beta) .
$$

Lemma 5.1. Suppose $\alpha$ and $\beta$ exist in the sense of Definition 5.1 and there exists a function $g \in L_{1}\left(I,[0, \infty)^{m}\right)$ such that for any $x, x^{\prime} \in \mathbb{R}^{m}$

$$
\left|f\left(t, X, x^{\prime}\right)\right| \leq g(t) \quad(a \leq t \leq b)
$$

where $X$ is defined in (9). Assume also that conditions (19) hold. Then problem (25) has a solution.

Proof. Define $f_{*}\left(t, x, x^{\prime}\right)$ as follows. We have for $i=1, \ldots, m, t \in I$ and $x, x^{\prime} \in \mathbb{R}^{m}$ that

$$
\begin{aligned}
& f_{* i}\left(t, x, x^{\prime}\right)= \\
& \quad\left\{\begin{array}{l}
f_{i}\left(t, X, x^{\prime}\right) \quad \text { if }-\left|x_{i}^{\prime}-\alpha_{i}^{\prime}(t)\right|+\alpha_{i}(t) \leq x_{i} \leq\left|x_{i}^{\prime}-\beta_{i}^{\prime}(t)\right|+\beta_{i}(t) \\
f_{i}\left(t, X, x_{1}^{\prime}, \ldots, x_{i-1}^{\prime}, \alpha_{i}^{\prime}(t), x_{i+1}^{\prime}, \ldots, x_{m}^{\prime}\right) \text { if } x_{i} \leq-2\left|x_{i}^{\prime}-\alpha_{i}^{\prime}(t)\right|+\alpha_{i}(t) \\
f_{i}\left(t, X, x_{1}^{\prime}, \ldots, x_{i-1}^{\prime}, \beta_{i}^{\prime}(t), x_{i+1}^{\prime}, \ldots, x_{m}^{\prime}\right) \quad \text { if } x_{i} \geq 2\left|x_{i}^{\prime}-\beta_{i}^{\prime}(t)\right|+\beta_{i}(t)
\end{array}\right.
\end{aligned}
$$

and $f_{* i}$ is linear in $\left(x, x^{\prime}\right)$ as

$$
\begin{aligned}
-2\left|x_{i}^{\prime}-\alpha_{i}^{\prime}(t)\right|+\alpha_{i}(t) & \leq x_{i} \leq-\left|x_{i}^{\prime}-\alpha_{i}^{\prime}(t)\right|+\alpha_{i}(t) \\
\left|x_{i}^{\prime}-\beta_{i}^{\prime}(t)\right|+\beta_{i}(t) & \leq x_{i} \leq 2\left|x_{i}^{\prime}-\beta_{i}^{\prime}(t)\right|+\beta_{i}(t) .
\end{aligned}
$$

For example, if $x_{i}^{\prime}-\beta_{i}^{\prime}(t)>0$ and $\left(x_{i}^{\prime}-\beta_{i}^{\prime}(t)\right)+\beta_{i}(t) \leq x_{i} \leq 2\left(x_{i}^{\prime}-\beta_{i}^{\prime}(t)\right)+\beta_{i}(t)$, then

$$
f_{* i}\left(t, x, x^{\prime}\right)=f_{i}\left(t, X_{\beta_{i}}, x_{1}^{\prime}, \ldots, x_{i-1}^{\prime},\left(2 x_{i}^{\prime}-\beta_{i}^{\prime}(t)\right)-\left(x_{i}-\beta_{i}(t), x_{i+1}^{\prime}, \ldots, x_{m}^{\prime}\right)\right.
$$

where $X_{\beta_{i}}$ is defined in (21). The boundary value problem

$$
\left.\begin{array}{rl}
x^{\prime \prime} & =f_{*}\left(t, x, x^{\prime}\right) \\
x(a) & =\delta\left(\alpha(a), \phi_{a}(x), \beta(a)\right) \\
x(b) & =\delta\left(\alpha(b), \phi_{b}(x), \beta(b)\right)
\end{array}\right\}
$$


has a solution $x$ since the homogeneous problem $x^{\prime \prime}=0, x(a)=x(b)=0$ has the trivial solution only. Let us show that $x$ solves problem (25).

First prove the estimate $x_{1} \geq \alpha_{1}$. Suppose the contrary is true. Then for the difference $u_{1}=\alpha_{1}-x_{1}$ a point $t_{1} \in(a, b)$ exists such that $u_{1}\left(t_{1}\right)=\max _{a \leq t \leq b} u_{1}(t)>0$ and $u_{1}(t)<u_{1}\left(t_{1}\right)$ for $t_{1}<t<b$. By inequality (24), there exists $u_{1}^{\prime}\left(t_{1}\right)$ and for $t$ close enough to $t_{1}$ the derivatives $u_{1}^{\prime}(t)$ are close to $u_{1}^{\prime}\left(t_{1}\right)$ (the existence and continuity of $\alpha^{\prime}(t)$ at any point of the interval $I$ follows from [12: Chapter 1, $\S \S 1$ and 2]). Then for $t_{2} \in\left(t_{1}, t_{1}+\varepsilon\right)$ with $\varepsilon>0$ sufficiently small one has

$$
u_{1}^{\prime}\left(t_{2}\right)-u_{1}^{\prime}\left(t_{1}\right) \geq \int_{t_{1}}^{t_{2}}\left(f_{1}\left(t, X_{\alpha_{1}}(t), X_{\alpha_{1}}^{\prime}(t)\right)-f_{1 *}\left(t, x(t), x^{\prime}(t)\right) d t=c .\right.
$$

It follows from $x_{1}\left(t_{1}\right)<\alpha_{1}\left(t_{1}\right)$ and $x_{1}^{\prime}\left(t_{1}\right)=\alpha_{1}^{\prime}\left(t_{1}\right)$ that for $t \in\left[t_{1}, t_{2}\right]$

$$
\begin{gathered}
x_{1}(t)<-2\left|x_{1}^{\prime}(t)-\alpha_{1}^{\prime}(t)\right|+\alpha_{1}(t) \\
f_{* 1}\left(t, x_{1}(t), x_{1}^{\prime}(t)\right)=f_{1}\left(t, X(t), X_{\alpha_{1}}^{\prime}(t)\right)=f_{1}\left(t, X_{\alpha_{1}}(t), X_{\alpha_{1}}^{\prime}(t)\right) .
\end{gathered}
$$

Hence $c=0$, which contradicts the definition of $t_{1}$. The rest of the inequalities $\alpha \leq x \leq$ $\beta$ can be obtained analogously

Definition 5.3 (Generalized Bernstein conditions). Functions $\alpha, \beta:[a, b] \rightarrow \mathbb{R}^{m}$ such that $\alpha \leq \beta$ will be called lower and upper functions with respect to $f$ and $N=$ $\left(N_{1}, \ldots, N_{m}\right)$ if

1) $-N \leq \alpha^{\prime}(t) \leq N$ and $-N \leq \beta^{\prime}(t) \leq N$ in $I$

2) $\alpha$ and $\beta$ satisfy the Lipschitz condition

3) for any $i=1, \ldots, m, t_{1} \in(a, b)$ and $t_{2} \in\left(t_{1}, b\right)$, in which first order derivatives exist, and any $x \in A C^{(1)}\left(I, \mathbb{R}^{m}\right)$ the inequalities

$$
\begin{aligned}
& \alpha_{i}^{\prime}\left(t_{2}\right)-\alpha_{i}^{\prime}\left(t_{1}\right) \geq \int_{t_{1}}^{t_{2}} f_{i}\left(t, X_{\alpha_{i}}(t), X_{\left(-N, \alpha_{i}, N\right)}^{\prime}(t)\right) d t \\
& \beta_{i}^{\prime}\left(t_{2}\right)-\beta_{i}^{\prime}\left(t_{1}\right) \leq \int_{t_{1}}^{t_{2}} f_{i}\left(t, X_{\beta_{i}}(t), X_{\left(-N, \beta_{i}, N\right)}^{\prime}(t)\right) d t
\end{aligned}
$$

hold, respectively, where $X_{\gamma_{i}}$ are defined in (21) and

$$
X_{\left(-N, \gamma_{i}, N\right)}^{\prime}=\left(\delta\left(-N_{1}, x_{1}^{\prime}(t), N_{1}\right), \ldots, \gamma_{i}^{\prime}(t), \ldots, \delta\left(-N_{m}, x_{m}^{\prime}(t), N_{m}\right)\right) .
$$

Definition 5.4. For a triple $(\alpha, \beta, N)$ a function $B: I \times[0, \infty)^{m} \rightarrow[0, \infty)^{m}$ is called a generalized Bernstein function if $B\left(t, y_{1}, \ldots, y_{m}\right)$ is non-decreasing in $y_{i}(i=1, \ldots, m)$ and for any $x \in A C^{(1)}\left(I, \mathbb{R}^{m}\right)$ the inequalities $\alpha \leq x \leq \beta$ and $\left|x^{\prime \prime}(t)\right| \leq B\left(t,\left|x^{\prime}(t)\right|\right) \quad(t \in$ $I)$ together imply $\left|x^{\prime}(t)\right| \leq N$ for any $t \in I$.

Theorem 5.2. Let $\alpha$ and $\beta$ be lower and upper functions with respect to $f$ and $N$, let $B$ be a generalized Bernstein function for $(\alpha, \beta, N)$ with

$$
\left|f\left(t, x, x^{\prime}\right)\right| \leq B\left(t,\left|x^{\prime}\right|\right) \quad\left(a \leq t \leq b ; \alpha \leq x \leq \beta ; x^{\prime} \in \mathbb{R}^{m}\right)
$$


and let conditions (19) be fulfilled. Then problem (25) is solvable.

Proof. Consider the modified equation

$$
x^{\prime \prime}=f\left(t, x, X^{\prime}\right)
$$

where $X_{i}^{\prime}=\delta\left(-N_{i}, x_{i}^{\prime}, N_{i}\right)$ and $X^{\prime}=\left(X_{1}^{\prime}, \ldots, X_{m}^{\prime}\right)$. Lower and upper functions $\alpha$ and $\beta$ with respect to $f$ and $N$ are lower and upper functions in the sense of Definition 5.2 for system (26). Its right side is bounded with respect to $x^{\prime}$ for $t \in I$ and

$$
\alpha \leq x \leq \beta \text {. }
$$

Then, by Lemma 5.1, equation (26) has a solution $x$ which satisfies the boundary conditions in (25) and estimate (27). To prove the theorem it suffices to show that $\left|x^{\prime}(t)\right| \leq N \quad(t \in I)$. But

$$
\left|x^{\prime \prime}(t)\right|=\left|f\left(t, x(t), X^{\prime}(t)\right)\right| \leq B\left(t,\left|X^{\prime}(t)\right|\right) \leq B\left(t,\left|x^{\prime}(t)\right|\right)
$$

in the interval $I$ and by the definition of $B$ this estimate holds

Theorem 5.3. Suppose that $\alpha$ and $\beta$ exist with respect to $f$ and $N$, conditions (19) are fulfilled, and assume in addition the existence of continuous functions $\Phi_{i}$ : $\left[-N_{i}, N_{i}\right] \rightarrow(0,+\infty) \quad(i=1, \ldots, m)$ such that

$$
\left|f_{i}\left(t, x, x^{\prime}\right)\right| \leq \Phi_{i}\left(\left|x_{i}^{\prime}\right|\right) \quad \text { for } \quad\left\{\begin{array}{l}
a \leq t \leq b \\
\alpha(t) \leq x \leq \beta(t) \\
-N \leq x^{\prime} \leq N
\end{array}\right.
$$

and

$$
\int_{\lambda_{i}}^{N_{i}} \frac{s d s}{\Phi_{i}(s)}>\max _{I} \beta_{i}(t)-\min _{I} \alpha_{i}(t)
$$

where $\lambda_{i}=\frac{1}{b-a} \max \left\{\left|\beta_{i}(a)-\alpha_{i}(b)\right|,\left|\beta_{i}(b)-\alpha_{i}(a)\right|\right\}$. Then problem (25) is solvable.

Proof. Consider the modified equation (26) along with the boundary conditions in (25). A solution $x$ to this problem exists, due to Lemma 5.1, and satisfies the estimate $\alpha \leq x \leq \beta$.

Let us prove that $\left|x^{\prime}(t)\right| \leq N$. Consider the first component $x_{1}^{\prime}$ (the proof for the remaining components $x_{i}^{\prime}$ is similar). There exists $t_{0} \in(a, b)$ such that $\left|x_{1}^{\prime}\left(t_{0}\right)\right| \leq \lambda_{1}$ since otherwise $x_{1}$ is not bounded by $\alpha_{1}$ and $\beta_{1}$. Suppose that $x_{1}^{\prime}\left(t_{1}\right)>N_{1}$ at some point $t_{1}$. Then, by continuity of $x_{1}^{\prime}$, there exist $t_{2} \in\left[t_{0}, t_{1}\right)$ and $t_{3} \in\left(t_{2}, t_{1}\right]$ such that $x_{1}^{\prime}\left(t_{2}\right)=\lambda_{1}, x_{1}^{\prime}\left(t_{3}\right)=N_{1}$ and $\lambda_{1} \leq x_{1}^{\prime}(t) \leq N_{1}$ for all $t \in\left[t_{2}, t_{3}\right]$. We have in the interval $\left[t_{2}, t_{3}\right]$

$$
\begin{aligned}
x_{1}^{\prime \prime}(t) & =f_{1}\left(t, x_{1}(t), \ldots, x_{m}(t), x_{1}^{\prime}(t), \delta\left(-N_{2}, x_{2}^{\prime}(t), N_{2}\right), \ldots, \delta\left({ }_{N}, x_{m}^{\prime}(t), N\right)\right) \\
& \leq \Phi_{1}\left(x_{1}^{\prime}(t)\right) .
\end{aligned}
$$

Then $\frac{x_{1}^{\prime}(t) x_{1}^{\prime \prime}(t)}{\Phi_{1}\left(x_{1}^{\prime}(t)\right)} \leq x_{1}^{\prime}(t)$ and

$$
\int_{t_{2}}^{t_{3}} \frac{x_{1}^{\prime}(t) x_{1}^{\prime \prime}(t)}{\Phi_{1}\left(x_{1}^{\prime}(t)\right)} d t=\int_{\lambda_{1}}^{N_{1}} \frac{s d s}{\Phi_{1}(s)} \leq x_{1}\left(t_{3}\right)-x_{1}\left(t_{2}\right) \leq \max _{I} \beta_{1}(t)-\min _{I} \alpha_{1}(t)
$$

which contradicts the definition of $N_{1}$. The other three cases can be considered analogously, repeating corresponding steps of the proof for the scalar case (for details one may consult [8] and [2: Chapter 1, §1.4])

Remark 5.1. Theorem 5.3 improves and generalizes [15: Theorem 3.1]. 


\section{Even order equation}

A particular case of system (4) with a monotone right side is an even order equation

$$
u^{(2 m)}=f\left(t, u, u^{\prime \prime}, \ldots, u^{(2 m-2)}\right)
$$

with $f$ satisfying the so-called mixed monotonicity condition $[13,16]$.

Definition 6.1. We say that a function $f\left(t, y_{1}, \ldots, y_{m}\right)$ is mixed monotonous for fixed $t \in I$ if $(-1)^{m} f\left(t, y_{1}, \ldots, y_{m}\right)$ is non-decreasing in the variables $y_{j}$ for $j$ odd and non-increasing in $y_{j}$ for $j$ even (thus $f$ is always non-increasing in $y_{m}$ and monotonicities in other variables alternate).

Consider equation (28) together with the boundary conditions

$$
\left.\begin{array}{l}
u^{(2 j-2)}(0)=A_{2 j-2} \\
u^{(2 j-2)}(1)=B_{2 j-2}
\end{array}\right\} \quad(j=1, \ldots, m)
$$

Introduce lower and upper functions $\alpha$ and $\beta$ following [16], but relaxing their differential properties.

Definition 6.2. We say that $\beta: I \rightarrow \mathbb{R}$ is an upper function for equation (28) if it is of class $C^{(2 m-2)}$, the derivative $\beta^{(2 m-2)}$ satisfies the Lipschitz condition, and for any $t_{1} \in(a, b)$ and $t_{2} \in\left(t_{1}, b\right)$, in which the derivative $\beta^{(2 m-1)}(t)$ exists, the inequality

$$
(-1)^{m}\left[\beta^{(2 m-1)}\left(t_{2}\right)-\beta^{(2 m-1)}\left(t_{1}\right)\right] \geq(-1)^{m} \int_{t_{1}}^{t_{2}} f\left(t, \beta(t), \ldots, \beta^{(2 m-2)}(t)\right) d t
$$

holds. The definition of a lower function $\alpha$ is analogous, with the opposite inequality sign.

Theorem 6.1. Suppose $f: I \times \mathbb{R}^{m} \rightarrow \mathbb{R}$ satisfies the Carathéodory conditions and is mixed monotonous. Let there exist lower and upper functions $\alpha$ and $\beta$ such that:

(E1) $(-1)^{(j-1)}\left[\beta^{(2 j-2)}(t)-\alpha^{(2 j-2)}(t)\right] \geq 0 \quad(j=1, \ldots, m)$.

(E2) $\left\{\begin{array}{l}(-1)^{(j-1)} \beta^{(2 j-2)}(a) \geq(-1)^{(j-1)} A_{2 j-2} \geq(-1)^{(j-1)} \alpha^{(2 j-2)}(a) \\ (-1)^{(j-1)} \beta^{(2 j-2)}(b) \geq(-1)^{(j-1)} B_{2 j-2} \geq(-1)^{(j-1)} \alpha^{(2 j-2)}(b)\end{array} \quad(j=1, \ldots, m)\right.$.

Then there exists a solution $u$ of problem (28) - (29) such that

$$
\left.\begin{array}{l}
(-1)^{(j-1)}\left[\beta^{(2 j-2)}(t)-u^{(2 j-2)}(t)\right] \geq 0 \\
(-1)^{(j-1)}\left[u^{(2 j-2)}(t)-\alpha^{(2 j-2)}(t)\right] \geq 0
\end{array}\right\} \quad(j=1, \ldots, m) .
$$

Proof. It follows by reduction to the monotonous system (4) and application of Theorem 2.1. To show how this scheme works consider the equation

$$
u^{(6)}=f\left(t, u, u^{\prime \prime}, u^{(4)}\right)
$$


along with the boundary conditions

$$
\begin{array}{lll}
u(a)=A_{0} & u^{\prime \prime}(a)=A_{1} & u^{(4)}(a)=A_{2} \\
u(b)=B_{0} & u^{\prime \prime}(b)=B_{1} & u^{(4)}(b)=B_{2} .
\end{array}
$$

Suppose that $f\left(t, y_{1}, y_{2}, y_{3}\right)$ is mixed monotonous, that is, $f$ is non-increasing in $y_{3}$ and $y_{1}$ and non-decreasing in $y_{2}$, for fixed $t \in I$. Suppose that lower and upper functions $\alpha$ and $\beta$ exist satisfying conditions (E1) and (E2) also, that is $\beta \geq \alpha, \beta^{\prime \prime} \leq \alpha^{\prime \prime}, \beta^{(4)} \geq \alpha^{(4)}$ and

$$
\begin{array}{lll}
\beta(a) \geq A_{0} \geq \alpha(a) & \beta^{\prime \prime}(a) \leq A_{1} \leq \alpha^{\prime \prime}(a) & \beta^{(4)}(a) \geq A_{2} \geq \alpha^{(4)}(a) \\
\beta(b) \geq B_{0} \geq \alpha(b) & \beta^{\prime \prime}(b) \leq B_{1} \leq \alpha^{\prime \prime}(b) & \beta^{(4)}(b) \geq B_{2} \geq \alpha^{(4)}(b) .
\end{array}
$$

We continue introducing variables $\left(y_{1}, y_{2}, y_{3}\right)=\left(-u, u^{\prime \prime},-u^{(4)}\right)$ and converting problem (28) - (29) into the system

$$
\left.\begin{array}{l}
y_{1}^{\prime \prime}=-u^{\prime \prime}=-y_{2} \\
y_{2}^{\prime \prime}=u^{(4)}=-\left(-u^{(4)}\right)=-y_{3} \\
y_{3}^{\prime \prime}=-u^{(6)}=-f\left(t, u, u^{\prime \prime}, u^{(4)}\right)=-f\left(t,-y_{1}, y_{2},-y_{3}\right)
\end{array}\right\}
$$

with

$$
\begin{array}{lll}
y_{1}(a)=-A_{0} & y_{2}(a)=A_{1} & y_{3}(a)=-A_{2} \\
y_{1}(b)=-B_{0} & y_{2}(b)=B_{1} & y_{3}(b)=-B_{2} .
\end{array}
$$

It is an easy matter to see that the vector right side of (30)

$$
\operatorname{col}\left(-y_{2},-y_{3},-f\left(t,-y_{1}, y_{2},-y_{3}\right)\right)
$$

is a non-increasing in the variables $y_{j}$ function and hence Theorem 2.1 is applicable. The lower function $\alpha$ of equation (28) generates an upper vector function $\left(-\alpha, \alpha^{\prime \prime},-\alpha^{(4)}\right)$ for system (30) and, similarly, the upper function $\beta$ of equation (28) generates a lower vector function $\left(-\beta, \beta^{\prime \prime},-\beta^{(4)}\right)$ for system (30)

\section{References}

[1] Adje, A.: Sur et Sous - Solutions Généralisées et Problèmes aux Limites du Second Ordre. Bull. Soc. Math. Bel., Sér. B, 42 (1990), 347 - 368.

[2] Bernfeld, S. and V. Lakshmikantham: An Introduction to Nonlinear Boundary Value Problems. New York: Acad. Press 1974.

[3] Cabada, A. and R. L. Pouso: Extremal solutions of strongly nonlinear discontinuous second order equations with nonlinear functional boundary conditions. Nonlin. Anal. 42 (2000), 1377 - 1396.

[4] De Coster, C. and P. Habets: Upper and lower solutions in the theory of ODE boundary value problems. In: Non linear analysis and boundary value problems for ordinary differential equations (CISM Courses and Lectures: Vol. 371). Wien - New York: Springer 1997, pp. $1-77$. 
[5] Fabry, C. and P. Habets: Upper and lower solutions for second order boundary value problems with nonlinear boundary conditions. Nonlin. Anal. 10 (1986), 985 - 1007.

[6] Gudkov, V. V.: On properties of solutions of second order differential equations (in Russian). Latvijskii Mat. Ezhegodnik (Latvian Math. Annual) 22 (1978), 3 - 10.

[7] Hartman, P.: Ordinary Differential Equations. New York: Wiley 1964.

[8] Jackson, L. K.: Subfunctions and second-order ordinary differential inequalities. Adv. Math. 2 (1968), $307-363$.

[9] Kiguradze, I. T.: On some singular boundary value problems for second order nonlinear ordinary differential equations (in Russian). Diff. uravn. (Diff. Equ.) 4 (1968), 1753 1773 .

[10] Kiguradze, I. T. and B. Puža: On some boundary value problems for a system of ordinary differential equations with singularities (in Russian). Diff. uravn. (Diff. Equ.) 12 (1976), $2139-2148$.

[11] Klokov, Yu. A. and N. A. Vasilyev: Foundations of the Theory of Boundary Value Problems for Ordinary Differential Equations (in Russian). Riga: Zinatne 1978.

[12] Lepin, A. J. and L. A. Lepin: Boundary Value Problems for Second Order Ordinary Differential Equations (in Russian). Riga: Zinatne 1988.

[13] Ma Ruyun, Zhang Jihui and Fu Shengmao: The method of upper and lower solutions for fourth-order two-point boundary value problems. J. Math. Anal. Appl. 215 (1997), 415 422.

[14] Ponomarev, V. D.: Existence in the simplest boundary value problem for the second-order differential equation (in Russian). Latvijskii Mat. Ezhegodnik (Latvian Math. Annual) 22 (1978), $69-74$.

[15] Sadyrbaev, F.: Nonlinear fourth-order two-point boundary value problems. Rocky Mount. J. Math. 25 (1995), $757-781$.

[16] Sedziwy, S.: Upper and lower solutions method for even order two point boundary value problems. Rocky Mount. J. Math. (to appear).

Received 20.07.2000; in revised form 10.03.2001 